\title{
In Search of Trapped Modes in the Single-Cell Cavity Prototype for CESR-B*
}

\author{
W. Hartung \\ Laboratory of Nuclear Studies, Cornell University, Ithaca, New York 14853 \\ E. Haebel \\ CERN, AT Division, CH-1211 Geneva 23, Switzerland
}

"Success has made a failure of our HOM."

S. O'Connor

\section{INTRODUCTION}

Plans for CESR-B, the proposed upgrade of the CESR $e^{+} e^{-}$storage ring to a $B$-factory, call for single-cell superconducting cavities with heavily damped higher-order modes (HOMs); $Q \leq 100$ is required for the dangerous modes in order to avoid multi-bunch instabilities [1]. The cavity is designed to enable all HOMs to propagate into the beam pipe, where they are damped by a layer of microwave-absorbing ferrite. Other "single-mode cavity" designs have been shown to have trapped modes, i.e. HOMs with low fields in the beam tube even though they are above cutoff $[2,3]$. Trapped modes in the CESR-B cavity would be poorly damped by the ferrite loads and might have excessively high $Q$ 's.

Several different approaches have been taken in assessing the effectiveness of the CESR-B cavity design: (i) the $Q$ 's of monopole and dipole modes have been measured in a full-size copper cavity with ferrite loads $[4,5]$; (ii) the $Q$ 's of monopole modes have been predicted using SEAFISH to model the cavity and loads [5]; (iii) the current-voltage method has been used to search for trapped monopole modes. The results of (iii) are discussed in this paper. An explanation of the current-voltage method and some recent improvements to it are also given. A more detailed treatment is given in a separate report [6].

\section{THE CURRENT-VOLTAGE METHOD}

The current-voltage method was developed to calculate damping using programs like SUPERFISH [7] or URMEL [8] that do not model power absorption. The method prcdicts the $Q$ of a cavity mode which can propagate energy into the beam tube or some other transmission line. The transmission line is assumed to be terminated with a matched load (the method can in principle handle a line terminated by an arbitrary impedance).

Consider a resonant cavity driven by a generator and damped by a load. A possible circuit model for this situation is shown in Figure 1a. A Thévenin equivalent circuit for the cavity, generator, couplers, and transmission lines is shown in Figure $1 \mathrm{~b}$. The power $P$ dissipated in the load resistor $R$ is

$$
P=\frac{1}{2} R \frac{\left|V_{t h}\right|^{2}}{\left|Z_{t h}+R\right|^{2}}
$$

\footnotetext{
* Work supported by the National Science Foundation, with supplementary support from U. S.-Japan collaboration.
}

(a)

3180593-007

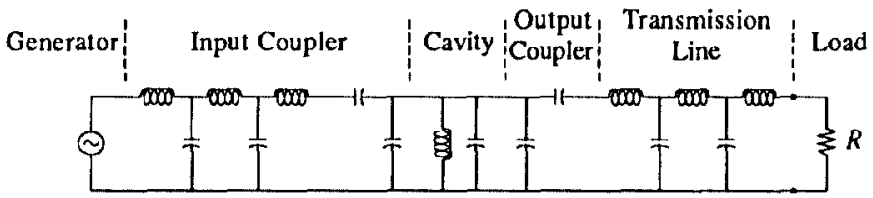

(b)

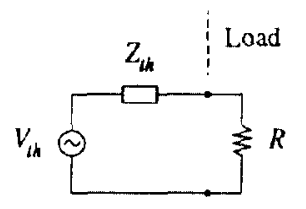

3180593-008

Figure 1. (a) A circuit model for a lossless resonant cavity excited by an ideal generator and damped by a resistive load at the end of a lossless transmission line; (b) the circuit model with the cavity, couplers, transmission line, and generator replaced by their Thévenin equivalent circuit.

$P$ cannot be calculated directly. If we replace $R$ by an open circuit and a short circuit in turn, however, we can express $P$ in terms of $V_{o p e n}$ and $I_{\text {short }}$. In the usual case, in which $R$ is real and $Z_{t h}$ is imaginary (meaning that the rest of the circuit has no resistive elements), the result is

$$
P=\frac{1}{2} R \frac{\left|V_{\text {open }}\right|^{2}}{\left|\frac{V_{\text {open }}}{I_{\text {ohort }}}\right|^{2}+R^{2}} .
$$

If the load is matched, then $R$ is equal to the characteristic impedance of the propagation mode. $V_{\text {open }}$ is determined from the transverse $\vec{E}$ field with an open boundary condition $\left(\vec{H}_{\|}=0\right)$ and $I_{s h o r t}$ is determined from the transverse $\vec{H}$ field with a short boundary condition $\left(\vec{E}_{\|}=0\right)$.

The voltage and current must be defined so that the power flow in a forward-travelling wave is $\frac{1}{2} V I^{*}$ (where $I^{*}$ is the complex conjugate of $I$ ) and the characteristic impedance of the line is $V / I$. Suitable definitions of $V$ and $I$ can be chosen for any waveguide or TEM transmission line ( $V$ and $I$ are uniquely defined only in a $T E M$ line). For the $T M_{01}$ mode in a circular waveguide, satisfactory definitions are

$$
V \equiv \sqrt{\pi} a E_{r}(r-a, \phi) ; \quad I \equiv \sqrt{\pi} a H_{\phi}(r=a, \phi),
$$

where $a$ is radius of the guide.

The procedure for using the method is then as follows: (i) Calculate the resonant modes of the cavity with the beam pipe terminated in an open and a short using a program like URMEL. (ii) Calculate $V_{\text {open }}$ and $I_{s h o r t}$ and 
deduce

$$
Q_{V} \equiv 2 \omega U R \frac{1}{\left|V_{\text {open }}\right|^{2}} ; \quad Q_{I} \equiv 2 \omega U \frac{1}{R} \frac{1}{\left|I_{\text {short }}\right|^{2}},
$$

where $\omega$ and $U$ are the calculated resonant angular frequency and stored energy, respectively. (iii) Identify the mode pairs that correspond to the same cavity mode with the opposite boundary condition in the beam tube. The coupling to the beam tube for that cavity mode is then

$$
Q_{\text {ext }}=Q_{V}+Q_{I} .
$$

$Q_{e x t}$ should be independent of the beam tube length $\ell$.

It should be noted that (i) a single mode of propagation is assumed, (ii) the frequency shift due to the open or short in the beam tube is neglected, and (iii) the energy stored in the transmission line is not subtracted. Because of (ii) and (iii), the method is most appropriate in the case of weak coupling to the beam tube.

\section{AN ORTHOGONALISED CURRENT-VOLTAGE METHOD}

There is one disadvantage to the current-voltage method as outlined above: the calculated $Q_{e x t}$ is influenced by the fields of other non-propagating or propagating modes. One must therefore choose $\ell$ large enough to eliminate contribution from evanescent fields. This also means that the method cannot be used if there is more than one mode of propagation. There is a remedy for these drawbacks, however: if the modes of propagation are orthogonal, the contribution to the calculated $Q_{\text {ext }}$ from the other modes of propagation can be eliminated using orthogonality.

If the modes of propagation are orthogonal, then for each mode $j$ of propagation, one can define transverse "unit fields" $\overrightarrow{\mathcal{E}}_{j}$ and $\overrightarrow{\mathcal{H}}_{j}$ which are proportional to the transverse fields in a forward-going wave; these unit fields must satisfy

$$
\int_{A} \overrightarrow{\mathcal{E}}_{j} \times \overrightarrow{\mathcal{H}}_{j^{\prime}}^{*} d a=\delta_{j j^{\prime}}
$$

where the integral is over the cross-section $A$ of the transmission line. Equation (3) is a necessary and sufficient orthogonality condition. The current $I_{j}$ and voltage $V_{j}$ for the $j$ th mode of propagation are obtained from the unit fields and the $\vec{E}$ and $\vec{H}$ fields calculated by URMEL or SUPERFISH as follows:

$$
V_{j}=\int_{A} \vec{E} \times \overrightarrow{\mathcal{H}}_{j}^{*} d a ; \quad I_{j}=\int_{A} \overrightarrow{\mathcal{E}}_{j}^{*} \times \vec{H} d a .
$$

The $Q_{\text {ext }}$ for the $j$ th mode of propagation is obtained from $V_{j}$ and $I_{j}$ using (1) and (2).

For the $T M_{0 n}$ mode in a circular waveguide, a suitable choice of unit fields gives the following expressions for the orthogonalised $V$ and $I$ :

$$
\begin{aligned}
V & =\frac{1}{\sqrt{\pi} a J_{1}\left(x_{0 n}\right)} \int_{0}^{2 \pi} \int_{0}^{a} E_{r}(r, \phi) J_{1}\left(\frac{x_{0 n}}{a} r\right) r d r d \phi \\
I & =\frac{1}{\sqrt{\pi} a J_{1}\left(x_{0 n}\right)} \int_{0}^{2 \pi} \int_{0}^{a} H_{\phi}(r, \phi) J_{1}\left(\frac{x_{0 n}}{a} r\right) r d r d \phi
\end{aligned}
$$

where $J_{m}(x)$ is the ordinary Bessel function of the first kind of order $m$ and $x_{0 n}$ is the $n$th zero of $J_{0}(x)$.

\section{COUPLING PREDICTIONS FOR THE CESR-B CAVITY}

$Q_{\text {ext }}$ calculations for $T M$ monopole modes in the CESRB cavity shape with a matched beam pipe were done with URMEL. Several different beam tube lengths were examined for "even" monopole modes (TMO-EE and TMO-EM in URMEL lingo); only one beam tube length was used for the "odd" monopoles (TM0-ME and TM0-MM). An even $T M_{012}$-like mode near $1.5 \mathrm{GHz}$ was sought, as the $T M_{012}$ has acquired a bad reputation in another single-cell cavity shape [2].

The calculated resonant frequencies of the first few even $T M$ monopoles are shown in Figure 2. There is a pair of modes at about $1.6 \mathrm{GHz}$ which remain close in frequency for all $\ell$; they also have lower fields in the beam tube than neighbouring modes. The calculated values of $Q_{V}$ and $Q_{I}$ for these two modes are shown in Figure 3a, along with the $Q_{\text {ext }}$ obtained with the hypothesis that they represent a single cavity mode. There is significant deviation for small $\ell$, but the $Q_{\text {ext }}$ converges to about 120 for large $\ell$. $Q_{V}, Q_{I}$, and $Q_{\text {ext }}$ values obtained using the orthogonalised

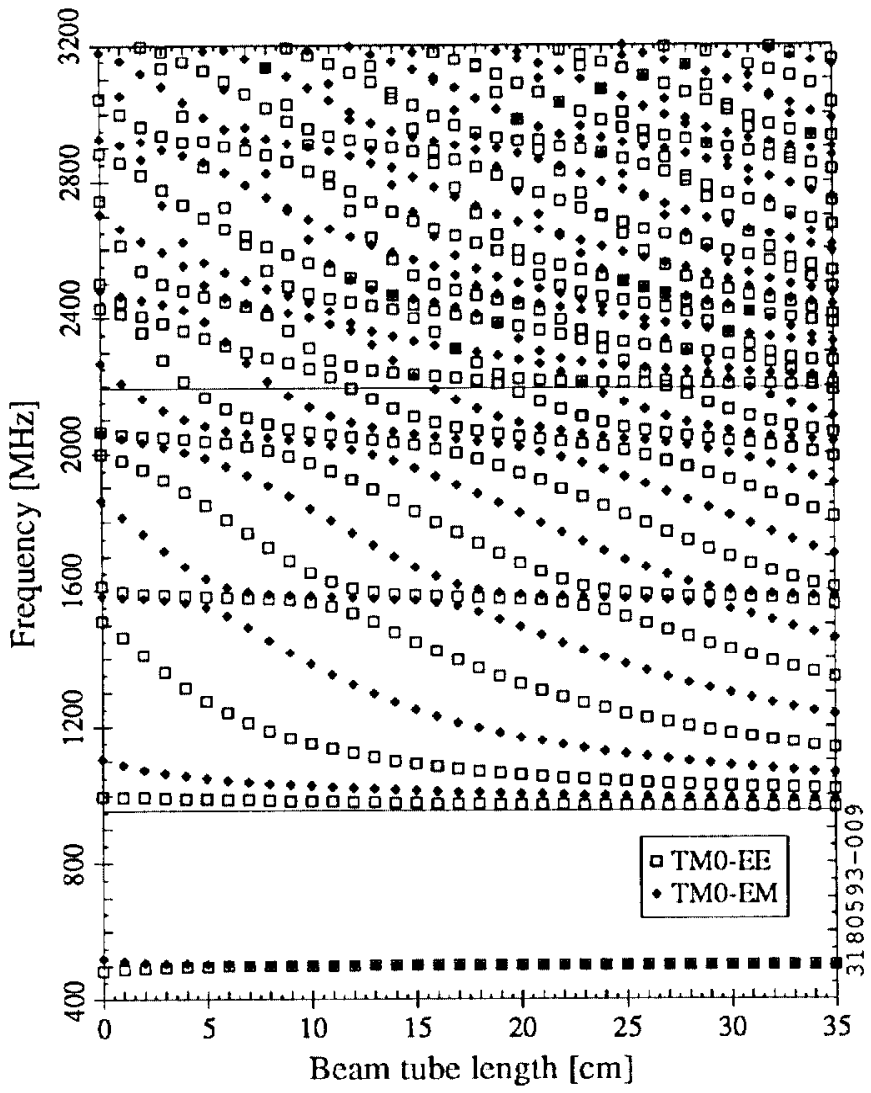

Figure 2. Dependence of the calculated resonant frequencies of even modes on $\ell$. The first two cutoff frequencies for $T M_{0}$ propagation are indicated by solid lines. The two modes at $500 \mathrm{MHz}$ are the cavity fundamental. 

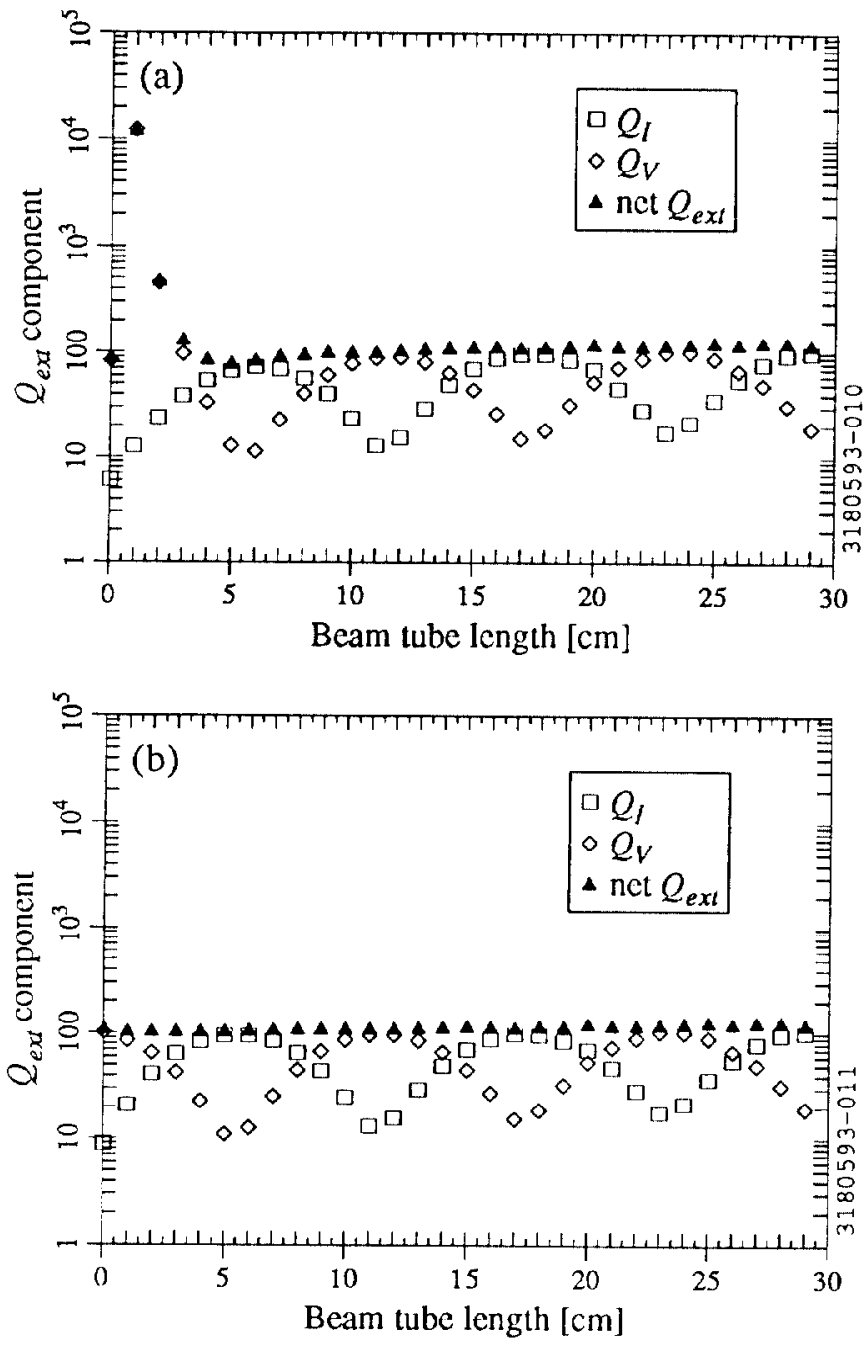

Figure 3. Dependence of the calculated $Q_{V}, Q_{I}$, and $Q_{\text {ext }}$ of the $1.6 \mathrm{GHz}$ modes on beam tube length (a) without and (b) with orthogonalisation. For (b), the values are based on $T M_{01}$ propagation.

method are shown in Figure $3 \mathrm{~b}$. The $Q_{\text {crt }}$ predicted with the orthogonalised method is almost constant even down to $\ell=0$.

The other modes were not paired up, so their $Q_{\text {ext }}$ cannot be predicted exactly, but it can be estimated from $Q_{V}$ and $Q_{I}$. Values of $Q_{V}$ and $Q_{I}$ calculated using the orthogonalised method with $\ell=20 \mathrm{~cm}$ are listed in Table 1 . From (2), we expect $Q_{\text {ext }} \leq 124$ for all of the modes listed.

Methods for calculating the shunt impedance $Z_{s}$ of these modes are still under study. The evidence suggests that $Z_{s} / Q \sim 0.1 \Omega$ for the $1.6 \mathrm{GHz}$ mode. The $Z_{s} / Q$ of modes with lower $Q$ 's may not be calculable with URMEL, because their resonant frequencies and field patterns are significantly affected by the boundary condition in the beam tube.
Table 1. $Q_{I}$ and $Q_{V}$ predicted with the orthogonalised method for $\ell=20 \mathrm{~cm}$. Modes above $T M_{01}$ cutoff and below $T M_{02}$ cutoff are listed.

\begin{tabular}{|c|c|c|c|}
\hline \multicolumn{2}{|c|}{ TMO-EE Modes } & \multicolumn{2}{|c|}{ TM0-EM Modes } \\
\hline $\begin{array}{c}\text { Frequency } \\
{[\mathrm{MHz}]}\end{array}$ & $Q_{I}$ & $\begin{array}{c}\text { Erequency } \\
{[\mathrm{MHz}]}\end{array}$ & $Q_{V}$ \\
\hline 973.9 & 26 & 1005.8 & 22 \\
\hline 1059.7 & 11 & 1170.3 & 7 \\
\hline 1326.8 & 6 & 1494.9 & 8 \\
\hline 1574.6 & 70 & 1589.0 & 54 \\
\hline 1681.5 & 9 & 1824.8 & 11 \\
\hline 1944.3 & 15 & 2018.2 & 35 \\
\hline 2049.2 & 61 & 2090.5 & 25 \\
\hline 2182.0 & 16 & & \\
\hline \multicolumn{2}{|c|}{ TM0-ME Modes } & \multicolumn{2}{|c|}{ TM0-MM Modes } \\
\hline $\begin{array}{c}\text { Frequency } \\
{[\mathrm{MHz}]}\end{array}$ & $Q_{I}$ & $\begin{array}{c}\text { Frequency } \\
{[\mathrm{MHz}]}\end{array}$ & $Q_{V}$ \\
\hline 972.3 & 27 & 1008.4 & 18 \\
\hline 1066.9 & 11 & 1165.8 & 8 \\
\hline 1289.7 & 8 & 1407.8 & 10 \\
\hline 1527.8 & 9 & 1677.0 & 8 \\
\hline 1833.4 & 11 & 1925.0 & 24 \\
\hline 1995.3 & 16 & 2135.8 & 10 \\
\hline
\end{tabular}

\section{CONCLUDING REMARKS}

A trapped monopole mode was found in the CESR-B cavity. Since its $Q$ is predicted to be 120 , we expect that it will not engender beam instabilities. The $Q$ 's predicted by the current-voltage method are of the same order as the $Q$ 's measured on the copper model and predicted by SEAFISH, which suggests that the ferrite layer is close to a matched load.

\section{REFERENCES}

[1] H. Padamsee et al., Conference Record of the 1991 IEEE Particle Accelerator Conference, p. 786-788.

[2] E. Haebel et al., CERN/EF/RF 84-1 (1984).

[3] S. Fornaca ct al., Proceedings of the 1987 IEEE Particle Accelerator Conference, p. 1818-1820.

[4] V. Veshcherevich et al., Proceedings of $B$ Factories: The State of the Art in Accelerators, Detectors, and Physics, SLAC-400/CONF-9204126, p. 177-180.

[5] D. Moffat et al., "Design, Fabrication and Testing of a Ferrite-lined HOM Load for CFSR-B," these proceedings.

[6] W. Hartung, NS/RF-92-1701, National Superconductor, 160000 Lincoln Avenue, Brentwood, Colorado 80523 (1992).

[7] K. Halbach \& R. F. Holsinger, Particle Accelerators 7, 213 (1976).

[8] T. Weiland, Nucl. Instrum. Methods 216, 329 (1983). 